\title{
Thomas de Padova: Alles wird Zahl: Wie sich die Mathematik in der Renaissance neu erfand
}

\author{
Carl Hanser Verlag, München 2021, 384 Seiten, ISBN \\ 978-3-446-26932-3/hbk; 978-3-446-27009-1/ebook
}

\section{Ilka Agricola}

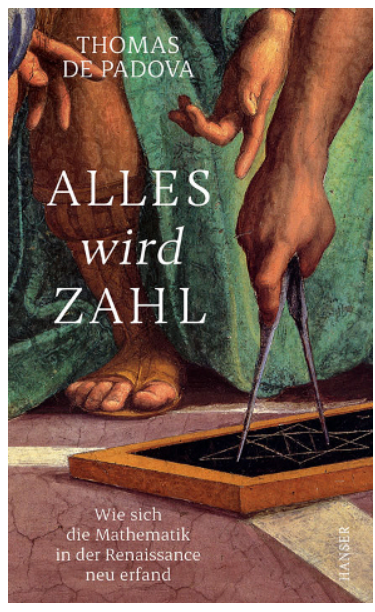
und historischen Kontext ein. So war der Sprung von seinen bisherigen Werken, der Physik, von Einstein, Leibniz und Newton hin zu den Renaissance-Mathematikern Regiomontanus, Stifel, Fibonacci und Cardano gar nicht weit. Herr de Padova schildert kenntnisreich, kurzweilig und doch instruktiv, wie die Gelehrten des 15. Jahrhunderts in einer Zeit des Umbruchs binnen weniger Generationen in der Mathematik einen Fortschritt erzielten, der seinesgleichen in der Fachgeschichte sucht. Die Begeisterung für die Antike, der sich sprunghaft entwickelnde Fernhandel, die

Ilka Agricola $(\bowtie)$

Fachbereich Mathematik und Informatik, Campus Lahnberge, Philipps-Universität Marburg,

35032 Marburg, Deutschland

E-Mail: agricola@mathematik.uni-marburg.de 
Erfindung des Buchdrucks und nicht zuletzt auch die Dynamik, die auf die Eroberung Konstantinopels durch die Osmanen 1453 folgte, sie alle bereiteten den Boden, auf dem die Mathematik vortrefflich gedeihen konnte. Nicht immer linear, wie man aus dem langen Nebeneinander von römischen und arabisch-indischen Ziffern lernt; aber doch wirkgewaltig. Die Erfindung der Variablen x, der Zeichen + und -, die erste mathematische Formelsprache -- dies sind Dinge, die uns von klein auf vertraut sind, deren Ursprung sich aber im Nebel der Zeiten verliert und über den wir nur wenig wissen - ein klares Versäumnis.

Der Autor arbeitet in seinem Buch deutlich heraus, dass der mathematische Abstraktionsschub dieser Zeit plötzlich eine Myriade von Anwendungen erlaubte, deren Nützlichkeit jedem sofort einleuchtete: Mathematik war nicht universell anwendbar OBWOHL, sondern gerade WEIL sie abstrakt war. Es ist eben egal, ob man den Dreisatz auf Getreidesäcke, Gestirne oder geometrische Figuren anwendet. Gleichzeitig erkennt man bei den Renaissance-Gelehrten eine fast kindliche Begeisterung für alles, was mit Mathematik zu tun hatte - Kalender und Sternentafeln, die noch Kolumbus benutzen sollte; Rechenwerke, die Rechenschulen in ganz Europa wie Pilze aus dem Boden sprießen ließen (man denke nur an Adam Ries!), die ersten gedruckten Auflagen der griechischen Klassiker, einige von ihnen gerade wiederentdeckt (Diophant) oder kaum bekannt (Apollonius von Perga). Die Zeit brachte Mathematik-Pioniere im Dutzend heraus, und die Kleinstaaterei in Italien und Deutschland erwies sich als günstig, gab es doch durch die Vielfalt der Zahlungsmittel, Kalender und Maßeinheiten immer etwas umzurechnen und obendrein eine erstaunliche Freiheit, sich da niederzulassen und zu wirken, wo man durch die Obrigkeit nicht zu sehr gestört wurde. Es war insgesamt eine wissenschaftsfreundliche Zeit, die leider mit den Glaubensstreitigkeiten, die bald ganz Europa von innen zerreissen würden, ihr Ende fand. So kam auch die Renaissance der Mathematik zu einem ersten Ende, und da endet auch dieses Buch. Doch das Wissen war in der Welt und nicht mehr aufzuhalten. Ein solch wissenschaftsfreundliches Klima würde man sich auch heute für uns wünschen! Dieses Buch zeigt uns, was dann alles möglich ist.

Es ist zu bedauern, dass in Schulen und Hochschulen nur wenig Zeit mit Mathematikgeschichte verbracht wird. Der in diesem Buch beschriebene Teil ist eher unbekannt, und so füllt das Buch von Thomas de Padova eine Lücke. Der Gegenstand ist breiten Leserschichten zugänglich, da hier noch keine „,höheren Mathematikkenntnisse" nötig sind, und trotzdem lernt man viel über abstraktes mathematisches Denken und Beweisen, den Innovationsschub, der von der Mathematik ausging, und die zu Unrecht vergessenen Akteure dieser stillen Revolution. So verwundert es nicht, dass das Buch im Herbst 2021 mit dem Medienpreis der DMV ausgezeichnet wurde.

Funding Open Access funding enabled and organized by Projekt DEAL.

Open Access Dieser Artikel wird unter der Creative Commons Namensnennung 4.0 International Lizenz veröffentlicht, welche die Nutzung, Vervielfältigung, Bearbeitung, Verbreitung und Wiedergabe in jeglichem Medium und Format erlaubt, sofern Sie den/die ursprünglichen Autor(en) und die Quelle ordnungsgemäß nennen, einen Link zur Creative Commons Lizenz beifügen und angeben, ob Änderungen vorgenommen wurden. 
Die in diesem Artikel enthaltenen Bilder und sonstiges Drittmaterial unterliegen ebenfalls der genannten Creative Commons Lizenz, sofern sich aus der Abbildungslegende nichts anderes ergibt. Sofern das betreffende Material nicht unter der genannten Creative Commons Lizenz steht und die betreffende Handlung nicht nach gesetzlichen Vorschriften erlaubt ist, ist für die oben aufgeführten Weiterverwendungen des Materials die Einwilligung des jeweiligen Rechteinhabers einzuholen.

Weitere Details zur Lizenz entnehmen Sie bitte der Lizenzinformation auf http://creativecommons.org/ licenses/by/4.0/deed.de. 